\title{
Al0.83Sc0.17N Contour-Mode Resonators with Electromechanical Coupling in Excess of 4.5\%
}

\author{
A. Lozzi, E. Ting-Ta Yen, P. Muralt and L. G. Villanueva
}

(C) 2018 IEEE. Personal use of this material is permitted. Permission from IEEE must be obtained for all other uses, in any current or future media, including reprinting/republishing this material for advertising or promotional purposes, creating new collective works, for resale or redistribution to servers or lists, or reuse of any copyrighted component of this work in other works. 


\title{
$\mathrm{Al}_{0.83} \mathrm{Sc}_{0.17} \mathrm{~N}$ Contour Mode Resonators with electromechanical coupling in excess of $4.5 \%$
}

\author{
Andrea Lozzi ${ }^{1 *}$, Ernest Ting-Ta Yen ${ }^{2}$, Paul Muralt ${ }^{3}$, and Luis Guillermo Villanueva ${ }^{1}$ \\ ${ }^{1}$ Advanced NEMS Lab, École Polytechnique Fédérale de Lausanne (EPFL), Lausanne, Switzerland \\ ${ }^{2}$ Kilby Labs, Texas Instruments Inc., Santa Clara, CA, USA \\ ${ }^{3}$ Ceramics Laboratory, École Polytechnique Fédérale de Lausanne (EPFL), Lausanne, Switzerland
}

"andrea.lozzi@epfl.ch

\begin{abstract}
In this paper we demonstrate the fabrication of contour mode resonators (CMRs) with $\mathrm{Al}_{0.83} \mathrm{Sc}_{0.17} \mathrm{~N}$ as piezoelectric layer. Moreover, we assess the electromechanical coupling and the maximum achieved quality factor from $150 \mathrm{MHz}$ to $500 \mathrm{MHz}$. In comparison to pure aluminum nitride (AIN) CMRs, our results show electromechanical coupling coefficients of more than a $2 \times$ factor higher at around $200 \mathrm{MHz}$. The highest quality factor is measured on a CMR operating at $388 \mathrm{MHz}$ and is in excess of 1600. From the characterization of devices operating at different frequencies material parameters of the $\mathrm{Al}_{0.83} \mathrm{Sc}_{0.17} \mathrm{~N}$ are extracted such as the stiffness constant, the relative permittivity and the piezoelectric constant. In particular, the reported $\mathrm{d}_{31}$ piezoelectric constant is equal to $-3.9 \mathrm{pm} / \mathrm{V}$. This represents a $2.25 \times$ improvement when compared to pure AIN. Finally, we report the first temperature compensation experimental results for $\mathrm{Al}_{0.83} \mathrm{Sc}_{0.17} \mathrm{~N}$ CMRs. Our results show that about $1.5 \mu \mathrm{m}$ of sputtered oxide, deposited on top of released resonator, allows near zero TCF for CMRs operating up to $500 \mathrm{MHz}$.
\end{abstract}

Index Terms-Contour mode resonators, aluminum scandium nitride, quality factor, electromechanical coupling, material properties, temperature compensation.

\section{INTRODUCTION}

In timing and filtering applications MicroElectroMechanical Systems (MEMS) based solutions are gathering attention for their small form factors and compatibility with CMOS technology. In particular, CMRs have been widely investigated for the possibility to set the operating frequency by layout design [1]. However, their figures of merit (FoM) still limit the competition with bulk acoustic wave resonators (BAW) and quartz crystals when used as timing or filtering solutions [2, 3].

Two key parameters for assessing the performance of an electroacoustic resonator are the quality factor (Q) and the electromechanical coupling $\left(\mathrm{k}_{\mathrm{t}}^{2}\right)$. High $\mathrm{Q}$ is desirable to achieve good close-in phase noise in oscillators [4] and sharp roll-off in filters, and in combination with $\mathrm{k}_{\mathrm{t}}^{2}$ to determine the insertion loss and maximum bandwidth of a filter $[5,6]$.

$\mathrm{Q}$ is defined as the ratio between the stored energy and the lost energy per cycle of vibration. Many dissipation mechanisms influence $Q$ such as air damping, electrical loading, acoustic losses, and material losses [7]. However, it has been shown that in pure aluminum nitride CMRs, the main dissipation mechanism is anchor loss up to $500 \mathrm{MHz}[8,9]$ and thermoelastic damping above $1 \mathrm{GHz}$ when comparing devices with equal area [10]. Many designs have been explored to minimize anchor loss: the anchors [8], the resonator shape [11, 12], the bus dimension [13], and the undercut created during release of the resonator $[14,15]$.

On the other hand, $\mathrm{k}_{\mathrm{t}}^{2}$ is a measure of the device efficiency in converting the electrical energy into mechanical energy, and then back to electrical. It has been shown that optimization of the layers' thickness with respect to the acoustic wavelength and the choice of the metal for the electrodes can improve $\mathrm{k}_{\mathrm{t}}^{2}$ in Lamb wave resonators [16]. In particular, in CMRs the optimal electroded area to maximize $\mathrm{k}_{\mathrm{t}}^{2}$ depends on the penetration depth of the electric field in the piezoelectric layer [1]. However, $\mathrm{k}_{\mathrm{t}}^{2}$ remains primarily limited by the relevant piezoelectric coefficient. Indeed, $\mathrm{k}_{\mathrm{t}}{ }^{2}$ is proportional to $\mathrm{d}_{31}{ }^{2}$, being $\mathrm{d}_{31}$ the piezoelectric constant involved in the energy conversion in CMRs. For this reason, many groups have studied piezoelectric materials that possess intrinsically higher piezoelectric constants. Studies have shown that lithium niobate $\left(\mathrm{LiNbO}_{3}\right)$ is a good candidate with $\mathrm{k}_{\mathrm{t}}^{2}$ up to $30 \%[6,17]$. However, the strong anisotropy of the material makes the fabrication very challenging and not compatible with standard IC processes.

On the other hand, alloying of the aluminum nitride (AIN) thin film has been explored for the advantage of being CMOS compatible. Moderate doping of the Al target with chrome, erbium, and tantalum [18-20] have proven to provide piezoelectric response enhancement up to $100 \%$. However, the most promising doping is with scandium (Sc). $50 \% \mathrm{Sc}$ replacement in the $\mathrm{Al}$ target led to a piezoelectric response improvement of around a factor of 4 [21]. This increase in electromechanical coupling is justified by the fact that Sc doping causes the Young's modulus of the Wurtzite structure to decrease, while the dielectric and piezoelectric constants increase [22].

$\mathrm{AlScN}$ has been used as piezoelectric layer to fabricate FBAR showing up to $100 \%$ increase in electromechanical coupling as the Sc doping is increased to 15\% [23]. A low-loss $3 \mathrm{GHz}$ surface acoustic wave (SAW) resonator showed relatively high $\mathrm{k}_{\mathrm{t}}^{2}$ of $5 \%$ in $46 \%$ doped AlScN [24]. Laterally vibrating Lamb wave resonators with up to $40 \%$ Sc doping achieved a 5x improvement in the electromechanical coupling with respect to pure AIN $[25,26]$.

In this study, we demonstrate $17.25 \%$ metal atom ratio AlScN one-port CMRs and we provide an extensive 
characterization of both material properties and device characteristics. In order to investigate if anchor loss is the main dissipation mechanism, similarly to AlN, devices with different anchor width $\left(\mathrm{W}_{\mathrm{a}}\right)$ are fabricated. Moreover, devices with different pitches are fabricated to assess the frequency variation as a function of the electrode pitches. Finally, we show that the same TCF (temperature coefficient of frequency variation) compensation technique used in AIN resonators (adding an oxide layer to the resonator) can be successfully implemented in AlScN.

\section{METHODS}

\section{A. Device and fabrication}

The devices fabricated in this work are all 1-port CMRs and the lateral field excitation (LFE) configuration is employed to electrically excite lateral motion in the resonators. In LFE, the bottom metal is a plate at floating potential, while the top metal is made of interdigitated (IDT) electrodes. The vertical component of the produced electric field is converted into lateral strain, in the direction of the resonator's width, by means of the piezoelectric coefficient $\mathrm{d}_{31}$.

An analytical formulation for the CMRs' resonance frequency is given by (1),

$f_{r}=v_{S 0} \cdot \frac{1}{\lambda}=\sqrt{\frac{E_{e q}}{\rho_{e q}}} \cdot \frac{1}{2 W}$

where $v_{s 0}$ is the stack's equivalent sound velocity associated with the $S O$ lateral mode, $\lambda$ is the acoustic wavelength, $E$ eq and $\rho e q$ are the equivalent modulus of elasticity and the density respectively, and $W$ is the electrode pitch.

The equivalent density and elastic modulus are weighted values by the thicknesses:

$\rho_{e q}=\frac{\sum_{1}^{n} \rho_{i} \cdot t_{i}}{\sum_{1}^{n} t_{i}} ; E_{e q}=\frac{\sum_{1}^{n} E_{i} \cdot t_{i}}{\sum_{1}^{n} t_{i}}$

where $\mathrm{n}$ is the number of different layers, and $t_{i}$ is the thickness of the $\mathrm{i}^{\text {th }}$ layer. Given the thickness ratios between piezoelectric and metals, the resonance frequency is mostly set by $W$. Unlike BAW devices [27], $f_{r}$ is therefore decoupled from the piezoelectric layer thickness, allowing fabrication of devices operating at different frequency on the same wafer.

The fabrication, schematically displayed in Fig. 1, starts with the patterning of the bottom Pt metal plate through lift-off onto high resistive silicon wafers $(>10 \mathrm{k} \mathrm{ohm} \cdot \mathrm{cm})$. Then, the piezoelectric layer is deposited via DC Magnetron sputtering using a $\mathrm{Al}_{0.83} \mathrm{Sc}_{0.17}$ target. The top metal $(\mathrm{Pt})$ is deposited and later patterned in interdigitated (IDT) electrodes via dry etching. In order to define the resonator shape in the AlScN, we first deposit a $1.5 \mu \mathrm{m}$ thick $\mathrm{SiO}_{2}$ hard mask, and we pattern it using an inductively coupled plasma (ICP) dry etcher using a standard recipe for $\mathrm{SiO} 2$ etching based on $\mathrm{C}_{4} \mathrm{~F}_{8}$ gas.

Subsequently, a Cl-based optimized recipe is used to pattern the $\mathrm{AlScN}$, allowing etching rates of about $100 \mathrm{~nm} / \mathrm{min}$. In Table 1. We include the six recipes that were tried, together with their etching rates. It can be noted that the substitution of Argon with $\mathrm{BCl}_{3}$ is beneficial to obtain high rates (Recipe 1-2 versus Recipe 3). Then, 1:1 ratio between $\mathrm{Cl}_{2}$ and $\mathrm{BCl}_{3}$ is found to provide the highest etching rate when using $\mathrm{Cl}_{2}$ and $\mathrm{BCl}_{3}$ chemistry (Recipe 3 versus Recipe 4-5). Finally, increasing the RF platen power provides a further enhancement of the etching rate (Recipe 3 versus Recipe 6). In particular, from $150 \mathrm{~W}$ to $200 \mathrm{~W}$ the etching rate increases $12 \%$. In this work, the RF platen is not further increased because higher powers lead to unstable plasma and thus unknown etching conditions. Even though the etching rate obtained with Recipe 6 is about $2 \times$ lower than the AIN etching rate in Cl-based ICP etchers, it allows successful etching of the AlScN piezoelectric layer with steep sidewall angle of $\sim 70^{\circ}$ (Fig. 3(b)). The final fabrication step comprises of the structure release in $\mathrm{SF}_{6}$. Successfully released CMRs are shown in Fig. 2 and Fig. 3.

\section{B. Characterization}

The devices are measured with a HP8719 Network Analyzer and scattering (S-) parameters are collected for each device. A standard short-load-open (SLO) calibration is performed before the measurements to shift the measurement's reference plane to the probe tips plane. Additionally, the device under test's (DUT) response is de-embedded using test structures without metallized faces and with shorted fingers [15] to subtract parasitics introduced by the pads and obtain an electrically unloaded (mechanical) quality factor $\left(\mathrm{Q}_{\mathrm{m}}\right)$ [28]. The Sparameters are then converted to admittance (Y-) parameters and the obtained response is fitted to a mBVD electrical model for parameters extraction [29].

To extract Q, the admittance response of a device, subtracted of the off-resonance background, is fitted to a Lorentzian curve. This method is equivalent to extracting $\mathrm{Q}$ using $-3 \mathrm{~dB}$ points in case of clean response. However, when spurious modes are present, especially in close proximity of the resonance, the Lorentzian fit is less affected and thus a better choice [30]. The electromechanical coupling is computed as the ratio of the motional capacitance $\left(\mathrm{C}_{\mathrm{m}}\right)$ and the static capacitance of the device $\left(\mathrm{C}_{0}\right)$ as in (3):

$k_{t}^{2}=\frac{\pi^{2}}{8} \frac{C_{m}}{C_{0}}$

\section{RESULTS AND DISCUSSIONS}

This section goes through the experimental results and is divided into four main sub-sections. The first section shows the evolution of $\mathrm{Q}$ as function of anchor width $\left(\mathrm{W}_{\mathrm{a}}\right)$ and bus dimension (B) (Fig. 2(a)) in devices with $\lambda=40 \mu \mathrm{m}$. The second part shows the dependence of $k_{t}^{2}$ on the frequency of the device. The third part displays how the electrical response of the measured CMRs is used to extract the material properties of AlScN. Finally, the Fourth section shows the experimental results concerning compensation of the temperature induced frequency shift using sputtered $\mathrm{SiO}_{2}$.

\section{A. Quality factor}

Devices with $\lambda=40 \mu \mathrm{m}(\mathrm{W}=20 \mu \mathrm{m})$ are fabricated sweeping two designs parameter, $\mathrm{Wa}$ and $\mathrm{B}$, to investigate their effect on $\mathrm{Q}$, similarly to what already done for pure AlN $[8,13] . \mathrm{W}_{\mathrm{a}}$ is 
swept from $0.25 \lambda$ to $0.9 \lambda$ in steps of $0.5 \lambda$. This fine step is chosen to detect potential sharp transition in Q. Regarding B, three dimensions are used: $0.15 \lambda, 0.2 \lambda$, and $0.25 \lambda$. The anchor length $\left(\mathrm{L}_{\mathrm{a}}\right)$ is fixed to $\lambda$.

Fig. 4(b) shows unloaded $\mathrm{Q}$ as function of $\mathrm{W}_{\mathrm{a}}$ for the three bus configurations in different colors. Each data point is the average of 3 identical devices.

The three bus configuration show similar trend. In particular, the maximum $\mathrm{Q}$ is always measured at $\mathrm{W}_{\mathrm{a}}=0.45 \lambda$. The peak is followed by a local minimum and subsequently, at $\mathrm{W}_{\mathrm{a}}=0.55 \lambda$, about $80 \%$ of the maximum Q is recovered. In contrast, the bus dimension does not change the trend but modulates the $\mathrm{Q}$ amplitude, having the highest average $\mathrm{Q}$ when the bus length is $\lambda / 4[13]$.

Even though the values of $Q$ are lower than what obtained in AlN, we observe that higher $Q$ are measured in devices at higher frequencies (Fig. 5(b)). In particular, the highest $\mathrm{Q}$ is measured for a device resonating at $388 \mathrm{MHz}$ (Fig. 5(a)) and is in excess of 1600 . We believe that a dependency of $Q$ in the resonator length (L) could explain the $\mathrm{Q}$ enhancement as the pitch is decreased. As a matter of fact, $\mathrm{L}$ is kept constant to $140 \mu \mathrm{m}$ for all the CMRs, which implies $\mathrm{L}=3.5 \lambda$ for a CMR working at 198 $\mathrm{MHz}$ and $\mathrm{L}=7 \lambda$ for a CMR operating at $388 \mathrm{MHz}$. Finally, the $\mathrm{Q}$ sensitivity to $\mathrm{W}_{\mathrm{a}}$, computed as $\left(Q_{\max }-Q_{\min }\right) /\left(Q_{\max }+\right.$ $Q_{\text {min }}$ ) on devices with identical $\mathrm{L}$ is $72 \%, 68 \%$, and $39 \%$ for devices operating at $151 \mathrm{MHz}, 194 \mathrm{MHz}$, and $388 \mathrm{MHz}$ respectively. This suggests that at higher frequencies the relative contribution of anchor dissipation is smaller. This is likely to be linked to the fact that the ratio L/lambda drops.

\section{B. Electromechanical coupling}

We also measure the electromechanical coupling of all devices, for different pitches. In the case of $\lambda=40 \mu \mathrm{m}$, the average $\mathrm{k}_{\mathrm{t}}^{2}$ of all the 126 measured devices is $4.42 \%$, representing a $2.2 \times$ improvement with respect to identical CMRs fabricated in AIN $\left(\mathrm{k}_{\mathrm{t}}^{2} \approx 2 \%\right)$. As an example, Fig. 4(a) shows the admittance response of a resonator operating at 194 $\mathrm{MHz}$ with the relative mBVD fit and parameters extraction.

After characterizing devices with different resonance frequencies (6 devices with highest $\mathrm{Q}$ for each frequency), we show that the average $\mathrm{k}_{\mathrm{t}}^{2}$ decreases as the frequency increases, reaching a minimum of $3.37 \%$ for the set of devices at $\sim 490$ $\mathrm{MHz}$ (Fig. 5(b)). After comparing our experimental results to FE simulations, we think that this degradation in electromechanical coupling is mainly due to two factors: electrode configuration and fringing field effect. (i) Electrode configuration: in order to maintain similar resonator width, the number of fingers was increased for higher frequencies up to 7 in the case of devices at $\sim 490 \mathrm{MHz}$. In this work, higher number of fingers resulted in more severe spurious modes in the admittance response. One possible explanation is that the acoustic mismatch between the regions with and without electrodes creates back scattered reflections that are seen as spurious modes in the admittance curve [31]. Moreover, when the resonator is wider, with respect to the wavelength, bending modes are more likely to couple into the electrode configuration. These unwanted peaks make the parameter extraction via fitting more difficult and result in an apparent degradation of the overall efficiency. (ii) Fringing field effect: since our electrode coverage was kept to $75 \%$ of the pitch across all the devices, the electrode separation decreases for larger frequencies and it reaches $2.5 \mu \mathrm{m}$ for the $479 \mathrm{MHz}$ devices is (about $2 \times$ the piezoelectric thickness). It was verified by FE simulations that fringing effect between adjacent electrodes happens and degrades $\mathrm{k}_{\mathrm{t}}^{2}$ for narrower pitches. This effect is more pronounced for $\mathrm{AlScN}$ than for AlN since the acoustic velocity of the former is smaller than the latter. However, the fringing effect does not seem to be the dominant factor, but rather a secondary player compared to the appearance of spurious modes. Importantly, our findings point towards a degradation of $\mathrm{k}_{\mathrm{t}}^{2}$ due mainly to spurious modes, rather than a degradation due to material properties, i.e. the piezoelectric coefficient of our AlScN is frequency independent.

\section{Material parameter extraction}

The starting point to extract the material parameters is to fit to (1) the resonant frequencies, as function of the pitch, of the ensemble of the fabricated CMRs, which in our case operate at 4 different frequencies (Fig. 6(b)). The devices have $\mathrm{W}=26 \mu \mathrm{m}$, $\mathrm{W}=20, \mathrm{~W}=10$, and $\mathrm{W}=8 \mu \mathrm{m}$ (Fig. 2 and Fig. 3) and they have $3,3,5$, and 7 fingers respectively in order to maintain similar resonator width and thus, simple simultaneous release. It is assumed that the density of AlScN is $3255 \mathrm{~kg} / \mathrm{m}^{3}$ [23], and the equivalent density $\left(\rho_{e q}\right)$ is given as an input parameter to a least square algorithm to extract the equivalent modulus of elasticity $\left(E_{e q}\right)$. Density and the Young modulus for Pt are $21450 \mathrm{~kg} / \mathrm{m}^{3}$ and $168 \mathrm{GPa}$, respectively.

As seen in (2), the thicknesses of the layers have to be known in order to properly compute the equivalents for the stack. For this reason, a cross section of the fabricated wafer is analyzed using scanning electron microscope (SEM). In Fig. 6(a), it can be seen that the bottom and top metal layers are about $100 \mathrm{~nm}$ thick while the piezoelectric layer is about $1.2 \mu \mathrm{m}$ thick. After obtaining $E_{e q},(2)$ is reversed to compute $E_{A l S c N}$ as below:

$E_{A l S c N}=\frac{E_{e q} \cdot T_{t o t}-E_{P t} \cdot T_{P t}}{T_{A l S c N}}$

In CMRs, the equivalent elasticity modulus can be assumed to be equal to $\mathrm{C}_{11}$, the stiffness component involved in the lateral expansion. The obtained $E_{e q}=C_{11}=339.8 \mathrm{GPa}$ closely matches the ab-initio calculations reported in literature for AlScN [32], confirming a softening (lower acoustic velocity) of the piezoelectric material with respect to AlN $(\approx$ $410 \mathrm{GPa}$ ). As an example to illustrate this difference, a device with $\mathrm{W}=20 \mu \mathrm{m}$, with the same thicknesses composing the stack, gives a resonance frequency of $194 \mathrm{MHz}$ for $\mathrm{AlScN}$ and 219 $\mathrm{MHz}$ for AlN.

Devices with $\mathrm{W}=20 \mu \mathrm{m}$ ( 3 fingers) with slightly different $\mathrm{L}$ are used to extract the relative permittivity of AlScN. Equation (5) is used to fit the measured static capacitance $C_{0}$ as function of resonator active length $\left(L_{\text {active }}=L-2 B\right)$. The bus is an inactive area because the bottom metal is removed by design underneath it. The relative permittivity is then computed as:

$\varepsilon_{r 33}=\frac{3}{2} \cdot C_{0} \cdot \frac{T_{\text {pze }}}{W_{e} \cdot L_{\text {active }}}$ 
where $T_{p z e}, W_{e} L_{\text {active }}$ are the piezoelectric thickness, the electrode coverage, and the active length of the resonator (Fig. (7)). As it is expected, $C_{0}$ has an increasing trend as the resonator is longer. The extracted $\varepsilon_{r 33}$ is 12.93 at $194 \mathrm{MHz}$. This value is in line with what previously reported for FBAR [23]. The small difference in $\varepsilon_{r 33}$ can be explained by the fact that in a CMR in LFE configuration, unlike for FBAR, the E lines are not entirely vertically distributed. Also, by comparing the measured and the numerically simulated (FEM) $\mathrm{C}_{0}$ values we verified that $\varepsilon_{r 33}$ can be assumed constant in this frequency range.

The electromechanical coupling $\mathrm{k}_{\mathrm{t}}^{2}$ can be expressed as follows:

$K^{2} \approx \frac{d_{31}^{2}}{s_{11}^{E} \cdot \varepsilon_{33}^{T}}=\frac{d_{31}^{2}}{\varepsilon_{0} \varepsilon_{r 33} / E_{e q}}$

where Eq. (6) is valid since the electrode's thickness is $<1 / 10$ of the piezoelectric layer's thickness.

If Eq. (6) is reversed, now that $E_{e q}$ and $\varepsilon_{r 33}$ are known and, given that the average $\mathrm{k}_{\mathrm{t}}^{2}$ is $4.42 \%$ (at $194 \mathrm{MHz}$ ) one can compute the piezoelectric $\left(d_{31}\right)$ coefficient for $\mathrm{Al}_{0.83} \mathrm{Sc}_{0.17} \mathrm{~N}$. The extracted $d_{31}$, at $194 \mathrm{MHz}$, is $-3.89 \mathrm{pm} / \mathrm{V}$, which represents a $2.25 \times$ improvement with respect to $\operatorname{AIN}\left(d_{31, A l N}=-1.73\right.$ $\mathrm{pm} / \mathrm{V})$. This extracted value is validated by excellent matching with ab-initio calculated value for $d_{31}(-3.87 \mathrm{pm} / \mathrm{V})$ [33].

\section{Temperature compensation}

MEMS-based oscillators enable low phase noise, low energy consumption and single-chip oscillator solutions [34, 35]. Among different technologies, AIN based MEMS resonators have larger power handling than $\mathrm{Si}$ based resonators and a greater $\mathrm{k}_{\mathrm{t}}^{2}$, which enables wider tuning range [36]. On the other hand, in AlN based MEMS there is no zero TCF orientation unlike in crystalline materials like quartz or Silicon [37]. The typical approach for TCF compensation in such MEMS relies on the use of $\mathrm{SiO}_{2}$ thin layers attached to the resonator stack $[38,39]$. Since $\mathrm{SiO}_{2}$ has the rare characteristic of a positive firstorder temperature coefficient of elasticity (TCE) [40], it can be used to compensate the negative TCF of the rest of the stack. In fact, people have used AlN-based resonators to demonstrate temperature sensors that operate linearly at different temperatures, depending on the amount of compensation [41].

In all of previous works, thermal oxide is used and it is often placed below the bottom metal. That is, the desired $\mathrm{SiO}_{2}$ thickness is analytically estimated a priori. Thus, a very fine analytical model and control of every thickness involved in the process is required.

Instead, we take a more flexible approach to easily obtain zero-TCF, using $\mathrm{SiO} 2$ compensation layer, in already fabricated devices: we sputter $\mathrm{SiO}_{2}$ on top of released resonators, using a stencil mask. The $\mathrm{SiO}_{2}$ thickness is checked with a mechanical profilometer in a region outside the resonator. This method allows simultaneous compensation of multiple frequencies and, at the same time, it does not require any a priori estimation of the needed thickness. As a consequence, after characterization of the TCF in a limited number of devices, the $\mathrm{SiO}_{2}$ thickness can be further adjusted without the need of fabricating a new wafer. The three sets of devices resonating at $194 \mathrm{MHz}(\mathrm{W}=20$ $\mu \mathrm{m}), 388 \mathrm{MHz}(\mathrm{W}=10 \mu \mathrm{m})$, and $479 \mathrm{MHz}(\mathrm{W}=8 \mu \mathrm{m})$ are characterized. Measurements are taken on a heated chuck, starting at $25^{\circ} \mathrm{C}$ up to $145^{\circ} \mathrm{C}$ with a sampling step of $30^{\circ} \mathrm{C}$.

In Fig. 8(a) the relative frequency variation as function of temperature is shown for devices operating at $194 \mathrm{MHz}$. The reference CMRs without oxide has a linear decreasing trend with a slope of $-27.6 \mathrm{ppm} /{ }^{\circ} \mathrm{C}$. By adding $\mathrm{SiO}_{2}$, the negative trend is mitigated. $1.5 \mu \mathrm{m}$ of $\mathrm{SiO}_{2}$ reduces the $1^{\text {st }}$ order TCF by a factor of 9. By adding thicker $\mathrm{SiO}_{2}$, a nearly zero TCF can be obtained. However, when the $\mathrm{SiO}_{2}$ thickness is larger than the resonator stack, the response in temperature becomes quadratic (i.e. relative frequency variation when $2.25 \mu \mathrm{m}$ of $\mathrm{SiO}_{2}$ is added). Table II report the $1^{\text {st }}$ and $2^{\text {nd }}$ order TCF as function of the $\mathrm{SiO}_{2}$ thickness for the three different frequencies. One can estimate the optimal $\mathrm{SiO}_{2}$ thickness looking at the $1^{\text {st }}$ order TCF, which is displayed in Fig. 8(b) for the three sets of devices. A linear fit is used to estimate the optimal $\mathrm{SiO}_{2}$ thickness to obtain a zero $1^{\text {st }}$ order TCF. CMRs operating at different frequencies require slightly different $\mathrm{SiO}_{2}$ thickness but always around $1.5 \mu \mathrm{m}$. For this thickness, the $2^{\text {nd }}$ order TCF is neglected since the quadratic term is negligible.

When targeting both a particular frequency and temperature compensation, one also needs to consider that adding $\mathrm{SiO}_{2}$, a softer material in the stack, causes a downshift of the resonant frequency. As it is seen in Eq. 1, the equivalent sound velocity depends on the thicknesses of all materials in the stack. In this study, the sputtered $\mathrm{SiO}_{2}$ leads to a $9 \%$ and $12 \%$ drop of the frequency when $1.5 \mu \mathrm{m}$ and $2.25 \mu \mathrm{m}$ of $\mathrm{SiO} 2$ are added respectively (Fig. 8(c)). Moreover, even though a full study of $\mathrm{Q}$ and $k_{t}^{2}$ dependency on $\mathrm{SiO}_{2}$ thickness is not conducted here, it is expected that the thicker the layer, the more degradation will be observed.

\section{CONCLUSIONS}

In this paper we demonstrate the fabrication of $\mathrm{Al}_{0.83} \mathrm{Sc}_{0.17} \mathrm{~N}$ CMRs. Our results show an improvement in the electromechanical coupling of more than $2 \times$ with respect to identical resonators in pure AlN, at around $200 \mathrm{MHz}$. A drop in $\mathrm{k}_{\mathrm{t}}{ }^{2}$ is observed at higher frequencies mainly due to the presence of spurious resonances. The highest Q, in excess of 1600, is obtained for a CMR operating at $388 \mathrm{MHz}$. Geometrical optimization, especially in the resonator length (i.e. scaling the resonator length according to $\lambda$ ), could improve $\mathrm{Q}$ in CMRs operating at lower frequencies. Moreover, as $\mathrm{AlScN}$ material parameters are extracted, modelling will open to a better understanding of the dissipation mechanisms for this new piezoelectric material.

From the electrical measurements several material properties are extracted. The extracted $\mathrm{C}_{11}=339.8 \mathrm{GPa}$ for $\mathrm{Al}_{0.83} \mathrm{Sc}_{0.17} \mathrm{~N}$ closely matches the ab-initio calculation present in literature [32]. Similarly, the relative permittivity is close to what has been reported for BAW [23]. Subsequently, the $d_{31}$ piezoelectric coefficient is computed. The extracted $\mathrm{d}_{31}$ value of $-3.89 \mathrm{pm} / \mathrm{V}$ matches the ab-initio prediction [33] and for devices operating around $200 \mathrm{MHz}$, corresponds to a $2.25 \times$ enhancement with respect to pure AIN. Finally, we report the experimental result of passive compensation of the frequency 
temperature drift using sputtered $\mathrm{SiO}_{2}$ on top of the CMRs. Differently from previous studies, in this work the $\mathrm{SiO}_{2}$ layer is added on top of already released resonators. This results in an unnecessary a priori accurate estimation of the compensation layer thickness and enables the measurement of the same resonator with different oxide thicknesses. We show that a $\mathrm{SiO}_{2}$ thickness around $1.5 \mu \mathrm{m}$ allows near zero TCF for devices operating up to $500 \mathrm{MHz}$, with a reasonable fabrication processing.

\section{ACKNOWLEDGEMENTS}

The authors acknowledge financial support from grant PP00P2 170590 of the Swiss SNF and from CMRs Radios project from Texas Instruments.

\section{REFERENCES}

[1] G. Piazza, P. J. Stephanou, and A. P. Pisano, "One and two port piezoelectric higher order contour-mode MEMS resonators for mechanical signal processing," Solid-State Electronics, vol. 51, pp. 1596-1608, Nov-Dec 2007.

[2] R. Ruby, R. Parker, and D. Feld, "Method of extracting unloaded Q applied across different resonator technologies," in Ultrasonics Symposium, 2008. IUS 2008. IEEE, 2008, pp. 1815-1818.

[3] C. C. Enz and A. Kaiser, MEMS-based circuits and systems for wireless communication: Springer Science \& Business Media, 2012.

[4] D. Lesson, "A simple model of feedback oscillator noise spectrum," proc. IEEE, vol. 54, pp. 329-330, 1966.

[5] C. Zuo, N. Sinha, M. B. Pisani, C. R. Perez, R. Mahameed, and G. Piazza, "12E-3 channel-select rf mems filters based on self-coupled aln contourmode piezoelectric resonators," in Ultrasonics Symposium, 2007. IEEE, 2007, pp. 1156-1159.

[6] S. Gong and G. Piazza, "Design and analysis of lithium-niobate-based high electromechanical coupling RF-MEMS resonators for wideband filtering," IEEE Transactions on Microwave Theory and Techniques, vol. 61, pp. 403-414, 2013.

[7] G. Piazza, P. J. Stephanou, and A. P. Pisano, "Piezoelectric aluminum nitride vibrating contour-mode MEMS resonators," Microelectromechanical Systems, Journal of, vol. 15, pp. 1406-1418, 2006.

[8] J. Segovia-Fernandez, M. Cremonesi, C. Cassella, A. Frangi, and G. Piazza, "Anchor Losses in AIN Contour Mode Resonators," Journal of Microelectromechanical Systems, vol. 24, pp. 265-275, Apr 2015.

[9] A. Lozzi, L. G. Villanueva, and E. T.-T. Yen, "Anchor Loss Dependence on Electrode Materials in Contour Mode Resonators."

[10] J. Segovia-Fernandez and G. Piazza, "Thermoelastic Damping in the Electrodes Determines \$ Q \$ of AlN Contour Mode Resonators," Journal of Microelectromechanical Systems, vol. 26, pp. 550-558, 2017.

[11] J. Zou, C.-M. Lin, G. Tang, and A. P. Pisano, "High-\$ $\{$ Q $\}$ ButterflyShaped AlN Lamb Wave Resonators," IEEE Electron Device Letters, vol. 38, pp. 1739-1742, 2017.

[12] C.-M. Lin, Y.-J. Lai, J.-C. Hsu, Y.-Y. Chen, D. G. Senesky, and A. P. Pisano, "High-Q aluminum nitride Lamb wave resonators with biconvex edges," Applied Physics Letters, vol. 99, p. 143501, 2011.

[13] C. Cassella, N. Singh, B. W. Soon, and G. Piazza, "Quality Factor Dependence on the Inactive Regions in AlN Contour-Mode Resonators," 20152015.

[14] B. Gibson, K. Qalandar, C. Cassella, G. Piazza, and K. L. Turner, "A Study on the Effects of Release Area on the Quality Factor of ContourMode Resonators by Laser Doppler Vibrometry," IEEE Transactions on Ultrasonics, Ferroelectrics, and Frequency Control, 2017.

[15] A. Lozzi, A. De Pastina, L. G. Villanueva, and E. T.-T. Yen, "Release area confinement in Contour mode resonators," in Ultrasonics Symposium (IUS), 2017 IEEE International, 2017, pp. 1-4.

[16] J. Zou, C.-M. Lin, C. Lam, and A. P. Pisano, "Transducer design for AlN Lamb wave resonators," Journal of Applied Physics, vol. 121, p. 154502, 2017.

[17] F. V. Pop, A. S. Kochhar, G. Vidal-Alvarez, and G. Piazza, "Laterally vibrating lithium niobate MEMS resonators with $30 \%$ electromechanical coupling coefficient," in Micro Electro Mechanical Systems (MEMS), 2017 IEEE 30th International Conference on, 2017, pp. 966-969.

[18] J. Luo, B. Fan, F. Zeng, and F. Pan, "Influence of Cr-doping on microstructure and piezoelectric response of AlN films," Journal of Physics D: Applied Physics, vol. 42, p. 235406, 2009.

[19] A. Kabulski, V. Pagán, and D. Korakakis, "Erbium Alloyed Aluminum Nitride Films for Piezoelectric Applications," MRS Online Proceedings Library Archive, vol. 1129, 2008.

[20] H. Liu, F. Zeng, G. Tang, and F. Pan, "Enhancement of piezoelectric response of diluted Ta doped AlN," Applied Surface Science, vol. 270, pp. 225-230, 2013.

[21] M. Akiyama, T. Kamohara, K. Kano, A. Teshigahara, Y. Takeuchi, and N. Kawahara, "Enhancement of piezoelectric response in scandium aluminum nitride alloy thin films prepared by dual reactive cosputtering," Advanced Materials, vol. 21, pp. 593-596, 2009.

[22] F. Tasnádi, B. Alling, C. Höglund, G. Wingqvist, J. Birch, L. Hultman, et al., "Origin of the anomalous piezoelectric response in wurtzite $\mathrm{Sc} \mathrm{x}$ Al 1-x N alloys," Physical review letters, vol. 104, p. 137601, 2010.

[23] R. Matloub, A. Artieda, C. Sandu, E. Milyutin, and P. Muralt, "Electromechanical properties of $\mathrm{Al} 0.9 \mathrm{Sc} 0.1 \mathrm{~N}$ thin films evaluated at $2.5 \mathrm{GHz}$ film bulk acoustic resonators," Applied Physics Letters, vol. 99, p. 092903, 2011

[24] K.-y. Hashimoto, S. Sato, A. Teshigahara, T. Nakamura, and K. Kano, "High-performance surface acoustic wave resonators in the 1 to $3 \mathrm{GHz}$ range using a ScAlN/6H-SiC structure," IEEE transactions on ultrasonics, ferroelectrics, and frequency control, vol. 60, pp. 637-642, 2013.

[25] A. Konno, M. Sumisaka, A. Teshigahara, K. Kano, K.-y. Hashimo, H. Hirano, et al., "ScAlN Lamb wave resonator in GHz range released by XeF 2 etching," in 2013 IEEE International Ultrasonics Symposium (IUS), 2013, pp. 1378-1381.

[26] L. Colombo, A. Kochhar, C. Xu, G. Piazza, S. Mishin, and Y. Oshmyansky, "Investigation of $20 \%$ scandium-doped aluminum nitride films for MEMS laterally vibrating resonators," in Ultrasonics Symposium (IUS), 2017 IEEE International, 2017, pp. 1-4.

[27] J. Rosenbaum, Bulk acoustic wave theory and devices: Artech House on Demand, 1988.

[28] M. Koolen, J. Geelen, and M. Versleijen, "An improved de-embedding technique for on-wafer high-frequency characterization," in Bipolar Circuits and Technology Meeting, 1991., Proceedings of the 1991, 1991, pp. 188-191.

[29] J. D. Larson, P. D. Bradley, S. Wartenberg, and R. C. Ruby, "Modified Butterworth-Van Dyke circuit for FBAR resonators and automated measurement system," in Ultrasonics Symposium, 2000 IEEE, 2000, pp. 863-868.

[30] S. Schmid, L. G. Villanueva, and M. L. Roukes, Fundamentals of nanomechanical resonators: Springer, 2016.

[31] J. Segovia-Fernandez, N.-K. Kuo, and G. Piazza, "Impact of metal electrodes on the figure of merit $(\mathrm{k} \mathrm{t} 2 \cdot \mathrm{Q})$ and spurious modes of contour mode AlN resonators," in Ultrasonics Symposium (IUS), 2012 IEEE International, 2012, pp. 299-302.

[32] S. Zhang, W. Fu, D. Holec, C. Humphreys, and M. Moram, "Elastic constants and critical thicknesses of ScGaN and ScAlN," Journal of applied physics, vol. 114, p. 243516, 2013.

[33] M. A. Caro, S. Zhang, T. Riekkinen, M. Ylilammi, M. A. Moram, O. Lopez-Acevedo, et al., "Piezoelectric coefficients and spontaneous polarization of ScAlN," Journal of Physics: Condensed Matter, vol. 27, p. 245901, 2015 .

[34] C. Cassella and G. Piazza, "Low Phase-Noise Autonomous Parametric Oscillator Based on a 226.7 MHz AlN Contour-Mode Resonator," Ieee Transactions on Ultrasonics Ferroelectrics and Frequency Control, vol. 62, pp. 617-624, Apr 2015.

[35] G. Wingqvist, L. Arapan, V. Yantchev, and I. Katardjiev, "A micromachined thermally compensated thin film Lamb wave resonator for frequency control and sensing applications," Journal of Micromechanics and Microengineering, vol. 19, p. 035018, 2009.

[36] V. Kaajakari, T. Mattila, A. Oja, and H. Seppa, "Nonlinear limits for single-crystal silicon microresonators," Journal of Microelectromechanical systems, vol. 13, pp. 715-724, 2004.

[37] A. Jaakkola, M. Prunnila, T. Pensala, J. Dekker, and P. Pekko, "Design rules for temperature compensated degenerately n-type-doped silicon MEMS resonators," Journal of Microelectromechanical Systems, vol. 24, pp. 1832-1839, 2015. 
[38] J. Zou, C.-M. Lin, Y.-Y. Chen, and A. P. Pisano, "Theoretical study of thermally stable $\mathrm{SiO} 2 / \mathrm{AlN} / \mathrm{SiO} 2 \mathrm{Lamb}$ wave resonators at high temperatures," Journal of Applied Physics, vol. 115, p. 094510, 2014.

[39] C.-M. Lin, T.-T. Yen, Y.-J. Lai, V. V. Felmetsger, M. A. Hopcroft, J. H. Kuypers, et al., "Temperature-compensated aluminum nitride Lamb wave resonators," IEEE transactions on ultrasonics, ferroelectrics, and frequency control, vol. 57, 2010.

[40] J. H. Kuypers, C.-M. Lin, G. Vigevani, and A. P. Pisano, "Intrinsic temperature compensation of aluminum nitride Lamb wave resonators for multiple-frequency references," in Frequency Control Symposium, 2008 IEEE International, 2008, pp. 240-249.

[41] C.-M. Lin, T.-T. Yen, V. V. Felmetsger, M. A. Hopcroft, J. H. Kuypers, and A. P. Pisano, "Thermally compensated aluminum nitride Lamb wave resonators for high temperature applications," Applied Physics Letters, vol. 97 , p. 083501,2010 . 


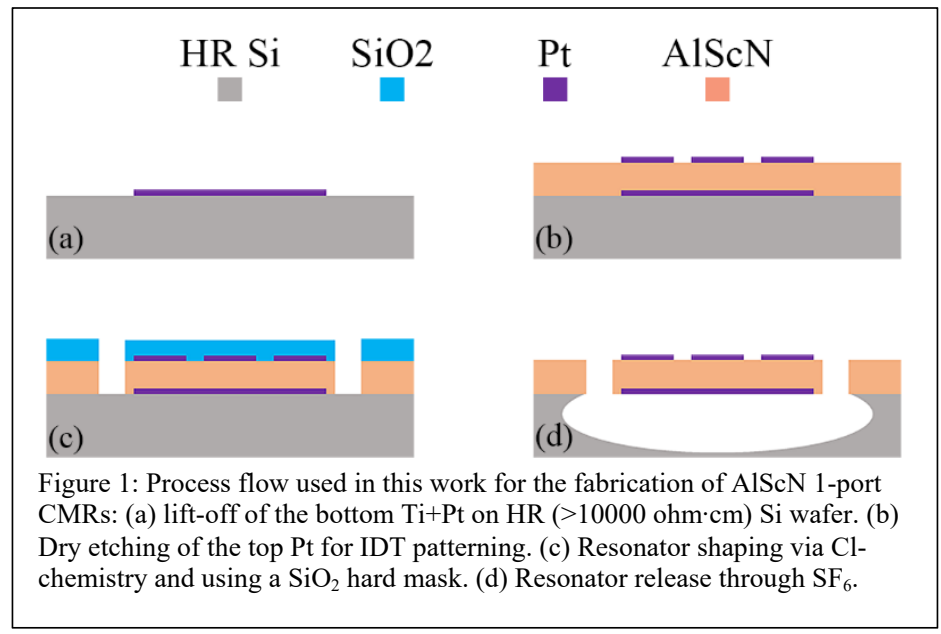

Table I: AlScN etching recipes and relative etching rates.

\begin{tabular}{c|c|c|c|c|c}
\multicolumn{2}{c}{$\begin{array}{c}\mathbf{C l}_{2} \\
{[\mathbf{s c c m}]}\end{array}$} & $\begin{array}{c}\mathbf{B C l}_{3} \\
{[\mathbf{s c c m}]}\end{array}$ & $\begin{array}{c}\text { Ar } \\
{[\mathbf{s c c m}]}\end{array}$ & $\begin{array}{c}\text { RF power } \\
{[\mathbf{W}]}\end{array}$ & $\begin{array}{c}\text { Etching rate } \\
{[\mathbf{n m} / \mathbf{m i n}]}\end{array}$ \\
\hline Recipe 1 & 20 & $/$ & 5 & 175 & 33 \\
\hline Recipe 2 & 20 & $/$ & 70 & 150 & 30 \\
\hline Recipe 3 & 10 & 10 & $/$ & 150 & 94 \\
\hline Recipe 4 & 20 & 10 & $/$ & 150 & 65 \\
\hline Recipe 5 & 10 & 20 & $/$ & 150 & 67 \\
\hline Recipe 6 & 10 & 10 & $/$ & 200 & 105 \\
& & & & &
\end{tabular}
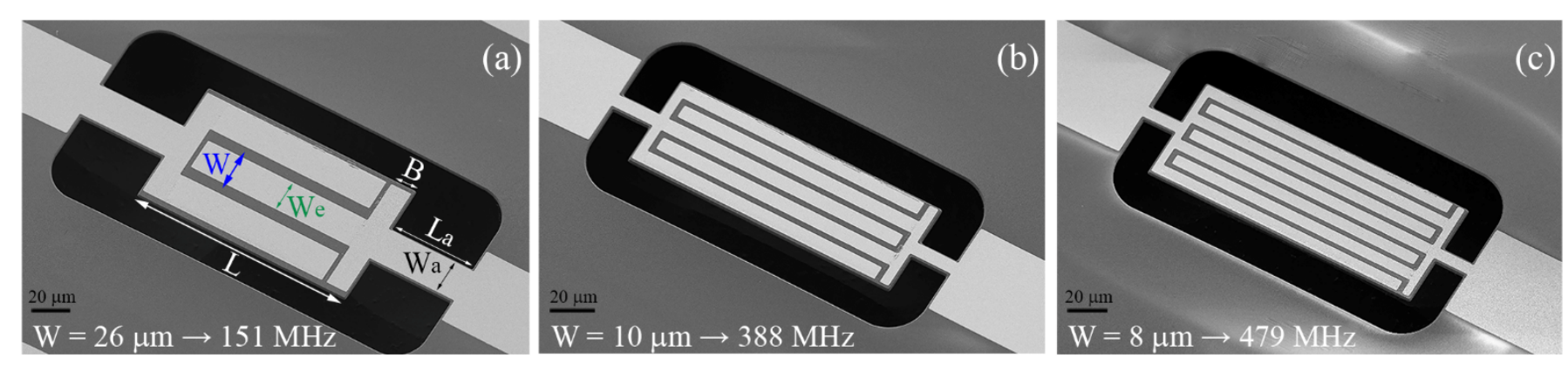

Figure 2: SEM pictures of three CMRs operating at different frequencies, obtained by lithographic tuning of W: $26 \mu \mathrm{m}$ (a), $10 \mu \mathrm{m}$ (b), and $8 \mu \mathrm{m}$ (c), which correspond to a resonant frequency of $150 \mathrm{MHz}, 388 \mathrm{MHz}$, and $479 \mathrm{MHz}$ respectively. In Fig. (a) all the designed parameters are displayed. 

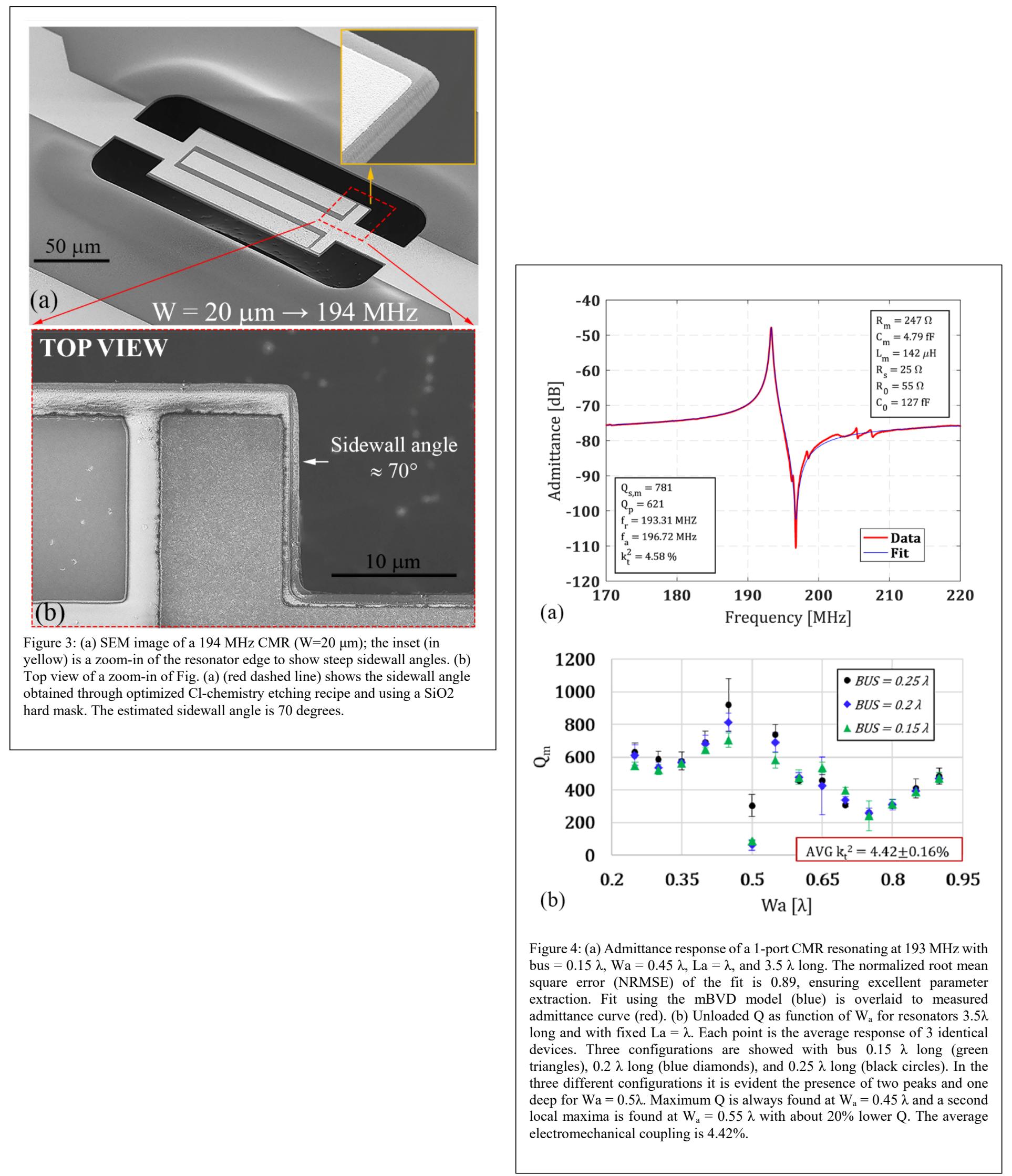


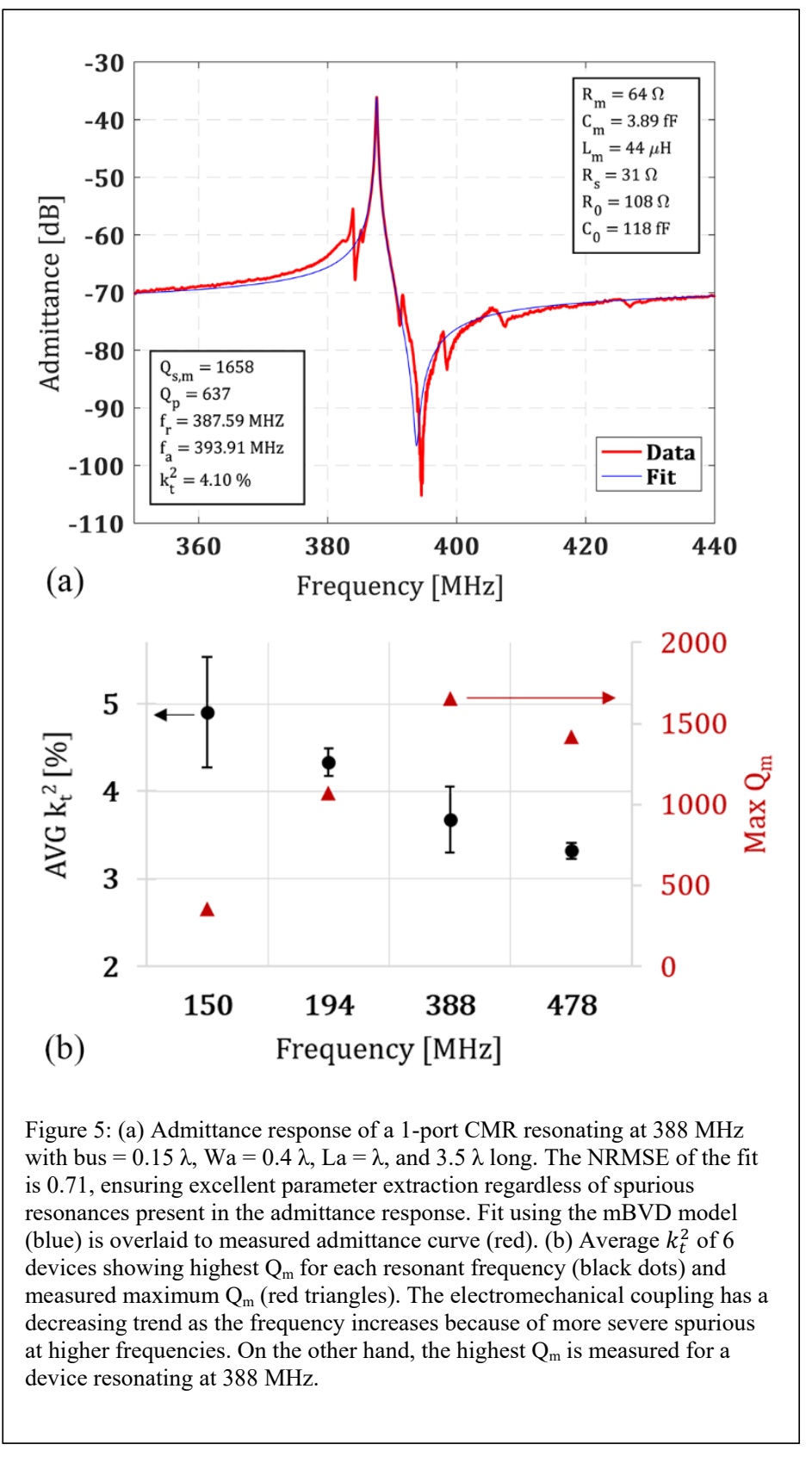

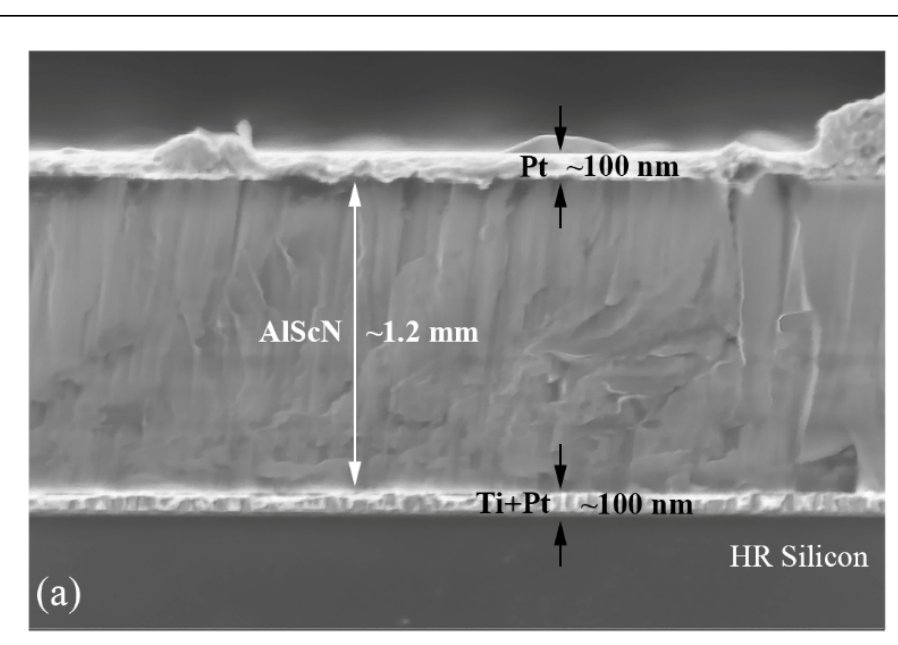

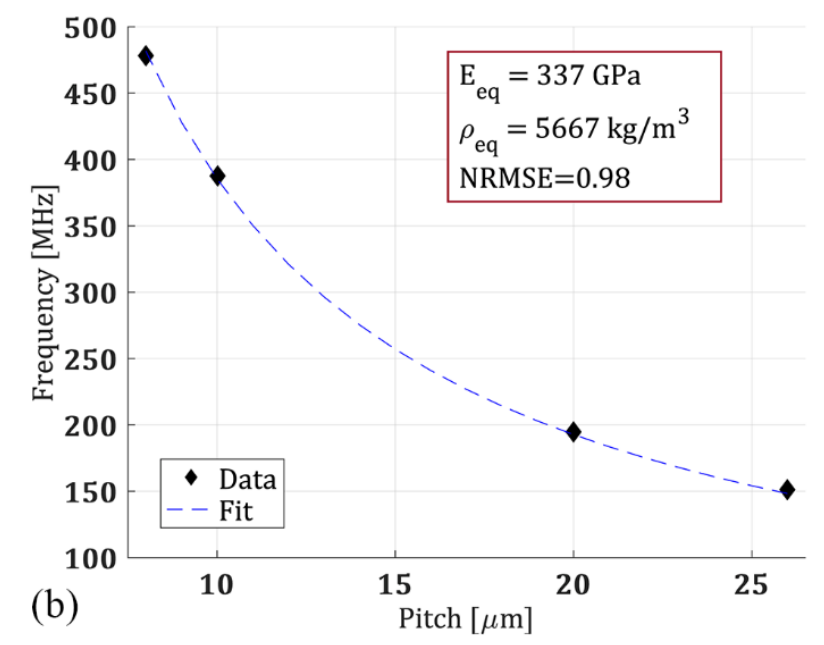

Figure 6: (a) Cross section of the fabricated wafer for assessment of the layers' thicknesses. The thickness of the top and the bottom metal is $100 \mathrm{~nm}$, while the piezoelectric layer thickness is $1.2 \mu \mathrm{m}$. (b) Measured resonant frequencies as a function of the pitches (black diamonds). Data are fitted, using a nonlinear square fitting algorithm to (1) to extract the equivalent modulus of elasticity of the whole stack. 

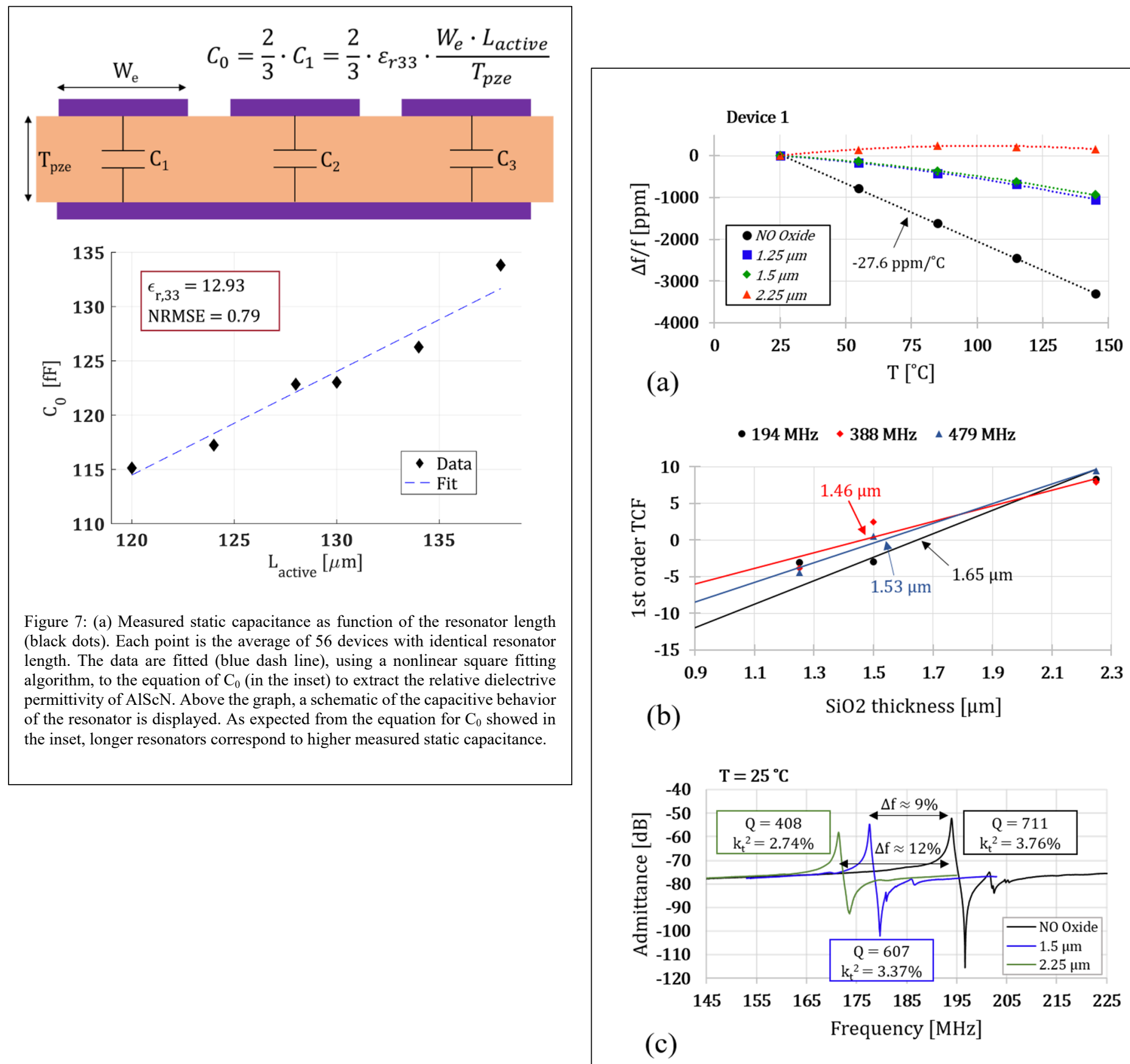

Figure 8: Fig. 8: (a) Relative frequency variation as a function of the substrate temperature. Each point is the average of 3 CMRs resonating at $194 \mathrm{MHz}$. In different colors are reported the measured relative frequency variations for different thickness of $\mathrm{SiO}_{2}$ deposited on the resonators: the reference with no oxide (black dots), $1.25 \mu \mathrm{m}$ of $\mathrm{SiO}_{2}$ (blue squares), $1.5 \mu \mathrm{m}$ (green diamonds), and $2.25 \mu \mathrm{m}$ (red triangles). It can be noted that $\mathrm{AlScN}$ has intrinsically a linear negative variation in frequency as temperature increases of -27.6 $\mathrm{ppm} / \mathrm{C}^{\circ}$. By adding $\mathrm{SiO}_{2}$ to the resonating stack, it is possible to reduce the negative slope, even though when the $\mathrm{SiO}_{2}$ thickness outweigh the stack thickness the linear trend is replaced by a quadratic response in temperature. (b) $1^{\text {st }}$ order TCF up to $145^{\circ} \mathrm{C}$. A zero TCF is obtained for different $\mathrm{SiO}_{2}$ thicknesses depending on the operating frequency of the resonator. Points are the experimentally extracted TCF, the lines are a linear interpolation to predict the zero $\mathrm{TCF} \mathrm{SiO}_{2}$ thickness. In particular, a zero $1^{\text {st }}$ order TCF is predicted at $1.65 \mu \mathrm{m}$ of $\mathrm{SiO}_{2}$ for devices resonating at $194 \mathrm{MHz}$ and for slightly thinner $\mathrm{SiO}_{2}$ thickness for devices resonating at $388 \mathrm{MHz}$ and $479 \mathrm{MHz}$.

(c) Admittance curve of a CMR operating at $194 \mathrm{MHz}$ without $\mathrm{SiO}_{2}$ (black), with $1.5 \mu \mathrm{m}$ of $\mathrm{SiO}_{2}$ (blue), and $2.25 \mu \mathrm{m}$ (green). The addition of $\mathrm{SiO}_{2}$ leads to a drop of $9 \%$ and of $12 \%$ in the resonant frequency for $1.5 \mu \mathrm{m}$ and $2.25 \mu \mathrm{m}$ of $\mathrm{SiO}_{2}$, respectively. 
Table II: $1^{\text {st }}$ and $2^{\text {nd }}$ order TCF for devices resonating at $194 \mathrm{MHz}, 388 \mathrm{MHz}$, and $479 \mathrm{MHz}$ in function of $\mathrm{SiO}_{2}$ thicknesses.

\begin{tabular}{|c|c|c|c|c|c|c|}
\hline \multirow[b]{3}{*}{ TCF } & \multicolumn{6}{|c|}{$1^{\text {st }}$ and $2^{\text {nd }}$ order TCF as function of $\mathrm{SiO}_{2}$ thickness } \\
\hline & \multicolumn{2}{|c|}{$194 \mathrm{MHz}$} & \multicolumn{2}{|c|}{$388 \mathrm{MHz}$} & \multicolumn{2}{|c|}{$479 \mathrm{MHz}$} \\
\hline & $\begin{array}{c}1 \text { 1st } \\
{\left[\mathrm{ppm}^{\circ}{ }^{\circ} \mathrm{C}\right]} \\
\end{array}$ & $\begin{array}{c}\text { 2nd } \\
{\left[\mathrm{ppb} /{ }^{\circ} \mathrm{C}^{2}\right]}\end{array}$ & $\begin{array}{c}1 \text { st } \\
{\left[\mathrm{ppm} /{ }^{\circ} \mathrm{C}\right]}\end{array}$ & $\begin{array}{c}\text { 2nd } \\
{\left[\mathrm{ppb} /{ }^{\circ} \mathrm{C}^{2}\right]}\end{array}$ & $\begin{array}{c}1 \text { st } \\
{\left[\mathrm{ppm} /{ }^{\circ} \mathrm{C}\right]}\end{array}$ & $\begin{array}{c}\text { 2nd } \\
{\left[\mathrm{ppb} /{ }^{\circ} \mathrm{C}^{2}\right]}\end{array}$ \\
\hline $\mathrm{No} \mathrm{SiO}_{2}$ & -27.60 & - & $N M$ & - & $N M$ & - \\
\hline $1.25 \mu \mathrm{m}$ & -3.09 & -33.2 & -3.81 & -38.3 & -4.47 & -32.2 \\
\hline $1.5 \mu \mathrm{m}$ & -3.02 & -28.6 & +2.48 & -52.8 & +0.53 & -39.6 \\
\hline $2.25 \mu \mathrm{m}$ & +8.29 & -41.2 & +7.88 & -28.7 & +9.94 & -23.6 \\
\hline
\end{tabular}

